\title{
The Stability Properties of a Coupled Pair of Non-Linear Partial Difference Equations
}

\begin{abstract}
By Bart J. Daly
1. Introduction. In applying finite difference techniques to the solution of partial differential equations, much concern is usually devoted to the stability properties of the resulting difference equations. The presence or lack of stability ordinarily can be determined by analysis and, if the equations are linear, the outcome determines their behavior for all time. If linear equations with constant coefficients are not stable, then any fluctuations present in the system will experience continuous growth in amplitude; but when non-linear equations are unstable, the rate of growth of fluctuations is subject to change. The non-linear terms may be of such a form as to counteract the instability and establish an upper limit to the amplitude of these fluctuations. When this is the case an equilibrium amplitude is eventually attained and henceforth only minor fluctuations about this level occur. The purpose of this paper is to study the mechanics by which this damping force is exerted, by investigating some of the phenomena observed when a certain coupled set of non-linear partial differential equations is differenced and run for thousands of cycles of calculation on the computer.

The interest in such a study is more than academic, for if it can be shown, for a particular set of difference equations, that instability fluctuations are bounded to a level which is small compared to the features of interest in a problem, then calculation with the unstable equations can be confidently pursued. Moreover, if one can obtain analytical expressions for the oscillation amplitudes in terms of the parameters of the system, one may then optimize those parameters in such a way as to minimize the effect of the oscillations.

Indeed, this study was motivated by the rather paradoxical success enjoyed by the Particle-in-Cell difference method [1] in representing high velocity fluid flow. In spite of the fact that the difference equations are unconditionally unstable, this success was achieved, in many problems, without recourse to the use of artificial dissipative terms. This paper discusses the underlying cause of the phenomenon with a simplified version of the Particle-in-Cell equations as a model. Greater elaboration, together with a discussion of application to the above-mentioned computing method, may be obtained from a somewhat more intensive report [2].

All calculations presented here were performed on an IBM Electronic Data Processing Machine, Type 704.
\end{abstract}

2. The Equations. The discussion will be concerned with the effect on the stability properties of the difference form of the one-dimensional wave equation caused by the addition of a single non-linear term. We write the wave equation in

Received ()ctober 22, 1962, revised April 22, 1963. Work performed under the auspices of the. I. S. Atomic Energy Commission. 
the coupled form

$$
\begin{aligned}
& \frac{\partial u}{\partial t}=-\frac{\partial v}{\partial x} \\
& \frac{\partial v}{\partial t}=-p^{2} \frac{\partial u}{\partial x},
\end{aligned}
$$

where $u$ and $v$ are related respectively to velocity and temperature, $x$ and $t$ are space and time coordinates and $p$ is a constant. The term to be added to these equations is one which arises naturally in the Particle-in-Cell finite difference method for fluid dynamics. The complete equations are

$$
\begin{aligned}
\frac{\partial u}{\partial t} & =-\frac{\partial v}{\partial x}+f \delta x \frac{\partial}{\partial x}\left(|u| \frac{\partial u}{\partial x}\right) \\
\frac{\partial v}{\partial t} & =-p^{2} \frac{\partial u}{\partial x},
\end{aligned}
$$

in which $f$ is a dimensionless constant of approximately unit magnitude and $\delta x$ is the finite difference space increment. They represent a simplified version of the equations which are solved by the Particle-in-Cell method. Notice that the added term is diffusive and non-linear; our principle object is to show its effect on the oscillations of the finite difference equations and, in particular, to show the manner in which these oscillations are bounded to small but finite amplitude.

Equations (2.2) are expressed in difference form as

$$
\begin{aligned}
\frac{u_{j}^{n+1}-u_{j}^{n}}{\delta t}= & -\frac{1}{2 \delta x}\left(v_{j+1}^{n}-v_{j-1}^{n}\right)+\frac{f}{2 \delta x}\left[\left|u_{j+1}^{n}+u_{j}^{n}\right|\left(u_{j+1}^{n}-u_{j}^{n}\right)\right. \\
& \left.-\left|u_{j}^{n}+u_{j-1}^{n}\right|\left(u_{j}^{n}-u_{j-1}^{n}\right)\right] \\
\frac{v_{j}^{n+1}-v_{j}^{n}}{\delta t}= & -\frac{p^{2}}{4 \delta x}\left(u_{j+1}^{n+1}+u_{j+1}^{n}-u_{j-1}^{n+1}-u_{j-1}^{n}\right) .
\end{aligned}
$$

The choice of time centering employed in this last equation is required in the original method to insure energy conservation. Its retention simplifies the study of these equations to some extent.

The finite difference system, over which these equations are defined, is composed of $N$ cells of equal length $\delta x$. The quantities $u$ and $v$ are defined at the center of each cell and the configuration is advanced in time, according to equations (2.3), by a sequence of time cycles, each of duration $\delta t$. The form of the difference equations (2.3) requires the consideration of two additional cells, $j=0$ and $j=N+1$, outside the system and the values of $u$ and $v$ at the center of these cells are defined by the boundary conditions on the system. The boundary condition on velocity requires that $u$ vanish at each end of the system and hence we require that $u_{0}=-u_{1}$ and $u_{N+1}=-u_{N}$.

A stability analysis of equations (2.3) indicates that they are unconditionally unstable, that is, any infinitesimal perturbation of the steady state conditions, $u_{j}=0, v_{j}=$ constant, gives rise to growing fluctuations. As these grow in amplitude, however, the non-linear term becomes important and establishes an upper bound 
(which depends upon the parameters of the system). Before analyzing the process we show in the next section some of the actual computer results which illustrate the features of interest.

3. Computer Experiments. A series of problems were run on the computer in order to test the effect of the various parameters on the final equilibrium conditions. An initial perturbation was supplied as a small velocity in each cell, and the resulting fluctuations were observed through their effect on the kinetic energy histories. In addition, the velocity profiles were analyzed in detail through Fourier decomposition.

Figure 1 shows the kinetic energy history for a series of machine runs in which the size of the system varies from 6 to 80 cells, all other parameters being fixed at the following values: $\delta t=0.25, \delta x=1.0, f=1.0$ and $p^{2}=0.4$. Boundary conditions specify $u=0$ at the ends of the region, and it can be shown that an exact solution of equation (2.2) at $t=\infty$ is $u=0$ everywhere. Thus the kinetic energy, $\frac{m}{2} \Sigma_{j} u_{j}{ }^{2}$, is a good measure of the non-vanishing fluctuation. Since the frequency of oscillations of the kinetic energy is too high to plot on the scale of Fig. 1, the curves trace the loci of the maximum and minimum points. The average midpoint between these lines at late times is taken as the equilibrium value.

Notice, in this figure, that fluctuations grow rapidly at first (as in a linear problem) but are eventually bounded to equilibrium. The amount of overshoot which precedes equilibrium, as well as the mean equilibrium amplitude, generally increase with the size of the system, although the 6 cell system is anomalous in both respects.

The machine results show that the course of any one calculation usually can be divided into three phases. In the first, or linear phase, the fluctuations grow rapidly in time. This phase is terminated by the achievement, in the mean, of velocity fluctuations large enough to give appreciable non-linear dissipation. In the second phase, there is a first order balance between the instability and the dissipation, but also there is a higher order imbalance leading to slow transition to final steady state (the third phase). The origin of these phases will be discussed in more detail in the following sections. Most of the calculations were not run long enough to show the Phase III behavior.

Other experiments were performed in which the time interval and the coefficient of the non-linear term were varied. It was found that the final equilibrium kinetic energy was proportional to $(\delta t / f)^{2}$, which behavior is explained in Section 4 .

The initial perturbation in these problems was such that only the symmetric (odd) Fourier modes of oscillation were originally present in the system. Furthermore the rate of growth of the even modes was observed to be very small compared to that of the odd modes, so that the nonsymmetric modes never contributed significantly to the energy of the system. A Fourier analysis of velocity profiles showed that, among the odd modes at late times, one mode of oscillation usually was dominant. When the number of cells in the system was small, the lowest frequency mode was predominant, exceeding the other modes in amplitude by several orders 


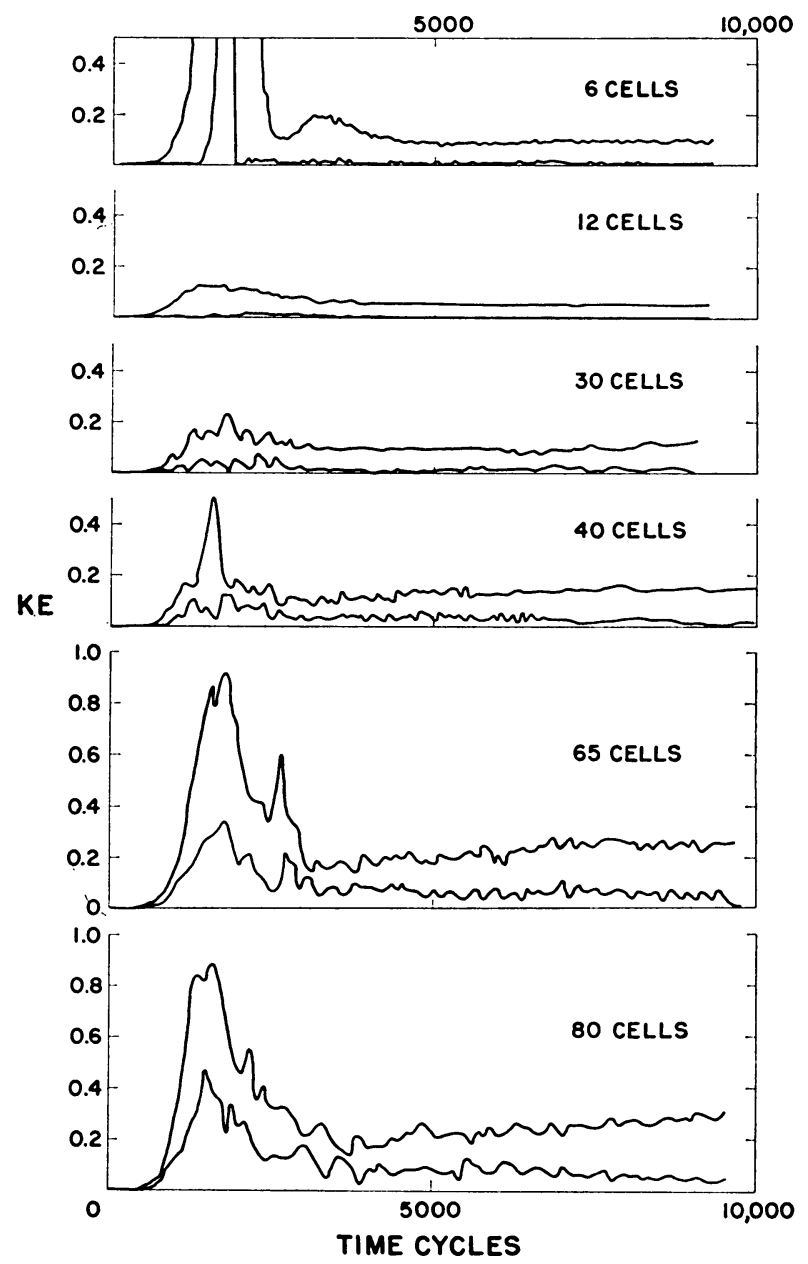

FIG. 1. Kinetic energy histories for various systems. The lines trace the loci of maximum and minimum values of kinetic energy.

of magnitude; but in larger systems one of the higher frequency modes generally dominated. In these latter cases the amplitude of the dominant mode was usually two or three times that of the next largest mode. Furthermore, although the wave number of the dominant mode differed from problem to problem, it was found that its late time amplitude was always nearly the same. These features are illustrated in Table 1.

4. The Mechanics of Dissipation. In the analysis of the problem of equilibrium balance, it will turn out to be sufficiently accurate to consider an approximation to the full equations. We therefore expand equations (2.3) in Taylor series about the center of the $j$ th cell and about time $n \delta t$. Neglecting terms higher than the first in $\delta x$ and $\delta t$, we obtain 


$$
\begin{aligned}
\frac{\partial u}{\partial t}+\frac{\delta t}{2} \frac{\partial^{2} u}{\partial t^{2}} & =-\frac{\partial v}{\partial x}+f \delta x \frac{\partial}{\partial x}\left(|u|^{2} \frac{\partial u}{\partial x}\right) \\
\frac{\partial v}{\partial t} & =-p^{2} \frac{\partial u}{\partial x},
\end{aligned}
$$

where, for convenience, subscripts and superscripts have been dropped

Now, to zero order

$$
\frac{\partial^{2} u}{\partial t^{2}}=p^{2} \frac{\partial^{2} u}{\partial x^{2}}
$$

and with this substitution equations (4.1) become

$$
\begin{aligned}
& \frac{\partial u}{\partial t}=-\frac{\partial v}{\partial x}+\frac{\partial}{\partial x}\left[\left(f \delta x|u|-\frac{1}{2} p^{2} \delta t\right) \frac{\partial u}{\partial x}\right] \\
& \frac{\partial v}{\partial t}=-p^{2} \frac{\partial u}{\partial x} .
\end{aligned}
$$

Notice that the diffusive effect in equations (4.3) depends upon the sign of the quantity

$$
\sigma(u) \equiv f \delta x|u|-\frac{1}{2} p^{2} \delta t .
$$

When $\sigma$ is positive the system is smoothed; when $\sigma$ is negative, fluctuation amplitudes increase. Hence, in this approximation, the mean amplitude of fluctuation occurs at $\sigma=0$, corresponding to

$$
|u|=\frac{p^{2} \delta t}{2 f \delta x}
$$

If we assume a probability distribution $P(u)$, of velocities about this mean, the corresponding mean kinetic energy for a system of $N$ cells of mass $m$ each is given by

$$
\overline{K E}=\frac{1}{2} N m \int_{-\infty}^{\infty} P(u) u^{2} d u
$$

For a normal distribution, $P(u)=.538 \gamma e^{-.91 \gamma^{2} u^{2}}, \gamma=\frac{f \delta x}{p^{2} \delta t}$; this gives

$$
\overline{K E}=.275 \frac{N m}{\gamma^{2}}=.275 N m\left[\frac{p^{2} \delta t}{f \delta x}\right]^{2} .
$$

In contrast, for a sharp distribution, in which $P(u)=\delta(u-\bar{u})$, we get

$$
\overline{K E}=\frac{1}{2} N m\left[\frac{p^{2} \delta t}{2 f \delta x}\right]^{2}=.125 N m\left[\frac{p^{2} \delta t}{f \delta x}\right]^{2} .
$$

A comparison of these predictions with computer results for typical values of the parameters is shown in Figure 2. The circles in the figure indicate observed values, while the upper line is a plot of relation (4.5), referring to normal distribution of velocities about the mean, and the lowest line shows the kinetic energy, equation (4.6), which would be attained if all cells had the mean velocity. It is seen that the 


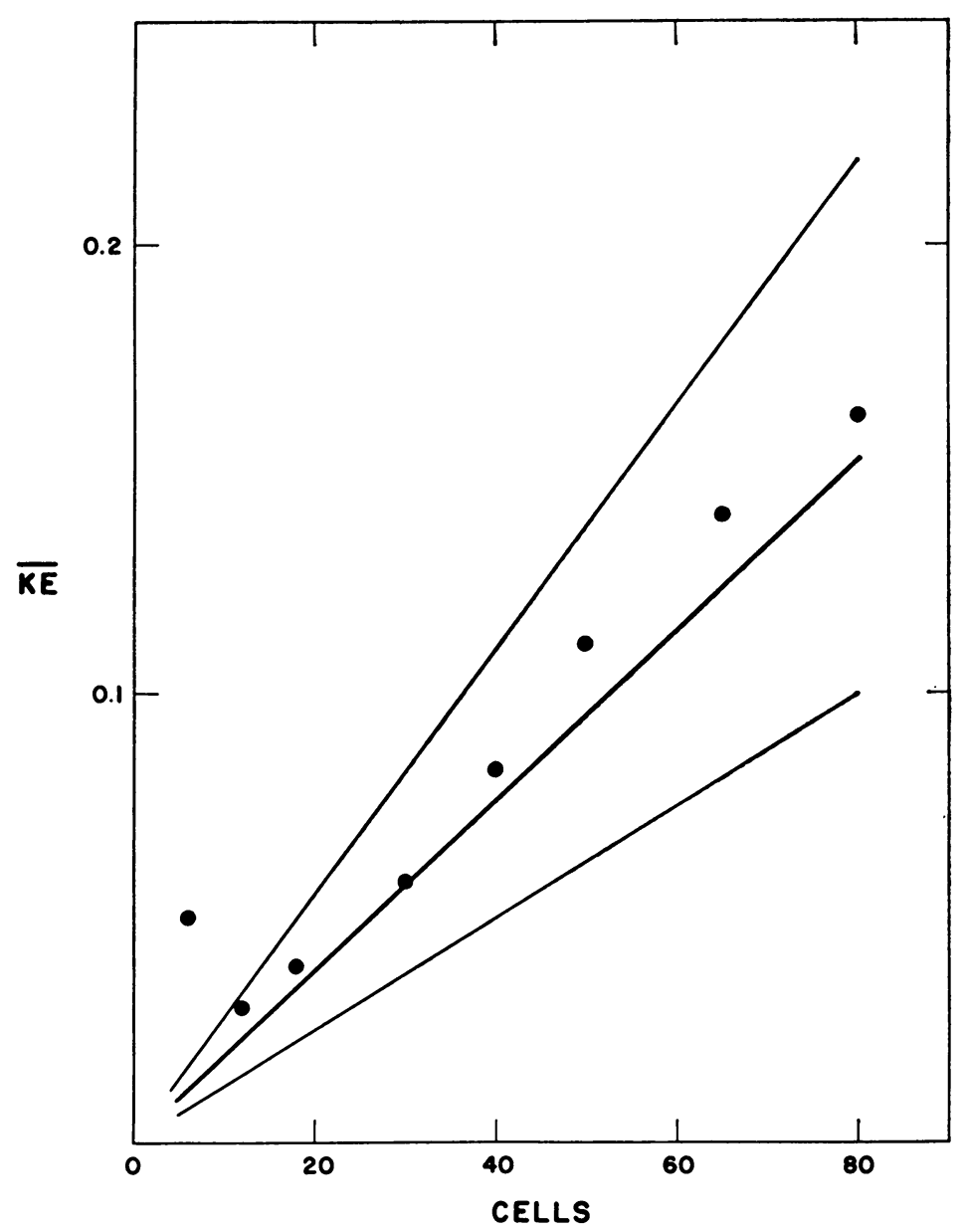

FIG. 2. A comparison of observed values of mean kinetic energy near final equilibrium with three predictions arrived at in the text.

actual distribution lies between these extremes. The prediction requires further analysis, the results of which are shown by the middle line in the figure and are derived in Section 5.

5. Modal Exchange of Energy. Thus far, the investigation, while shedding some light on the mechanics of the damping process and providing an estimate of the equilibrium amplitude, has told nothing about the normal modes of oscillation in the system or of the energy sharing between these modes. For this purpose we need a more detailed study of the difference equations.

Consider again equations (4.1). Following Kryloff and Bogoliuboff [3], we will assume that the solution of these first order equations does not differ much from the solution of the corresponding zero order equations (equations (2.1)), and will account for the difference by allowing the amplitude and phase of the zero order solution to vary with time. An appropriate solution of the zero order 
equations is

$$
\begin{aligned}
& u=A \sin k x \sin (\omega t+\varphi) \\
& v=p A \cos k x \cos (\omega t+\varphi),
\end{aligned}
$$

where $\omega=p k$. Application of the boundary conditions that $u$ shall vanish at each end of the system, determines the unique values which $k$ may assume,

$$
k=\frac{n \pi}{L}, \quad n=1,2, \cdots
$$

where $L=N \delta x$. Thus the complete solution of the zero order equations can be written

$$
\begin{aligned}
& u=\sum_{n=1}^{\infty} A_{n} \sin \frac{n \pi x}{L} \sin Q_{n} \\
& v=p \sum_{n=1}^{\infty} A_{n} \cos \frac{n \pi x}{L} \cos Q_{n},
\end{aligned}
$$

where $Q_{n}=\frac{n \pi p t}{L}+\varphi_{n}$, and the $A_{n}$ and $\varphi_{n}$ are constants determined by the initial conditions.

Now, considering $A_{n}$ and $\varphi_{n}$ as functions of time, we have

$$
\frac{\partial u}{\partial t}=\sum_{n}\left[\sin \frac{n \pi x}{L}\left(\dot{A}_{n} \sin Q_{n}+A_{n} \frac{n \pi p}{L} \cos Q_{n}+A_{n} \dot{\varphi}_{n} \cos Q_{n}\right)\right]
$$

so that, to impose the zero order solution, it is required that

$$
\dot{A}_{n} \sin Q_{n}+A_{n} \dot{\varphi}_{n} \cos Q_{n}=0 \text {. }
$$

Substitution of solution (5.2), subject to condition (5.3), into equations (4.1) gives

$$
\begin{aligned}
\sum_{n}\left[\frac{n \pi p \delta t}{2 L}\left(\frac{\dot{A}_{n}}{\cos Q_{n}}-A_{n} \frac{n \pi p}{L} \sin Q_{n}\right) \sin \frac{n \pi x}{L}\right] \\
=f \delta x \frac{\partial}{\partial x}\left\{\left|\sum_{n} A_{n} \sin \frac{n \pi x}{L} \sin Q_{n}\right| \sum_{m}\left[A_{m} \frac{m \pi}{L} \cos \frac{m \pi x}{L} \sin Q_{m}\right]\right\}
\end{aligned}
$$

plus an identity equation. Thus

$$
\begin{aligned}
& \frac{n \pi p \delta t}{4} {\left[\frac{\dot{A}_{n}}{\cos Q_{n}}-A_{n} \frac{n \pi p}{L} \sin Q_{n}\right] } \\
& \quad=f \delta x \int_{0}^{L} \sin \frac{n \pi x}{L} \frac{\partial}{\partial x}\left\{\left|\sum_{l} A_{l} \sin \frac{l \pi x}{L} \sin Q_{l}\right| \sum_{m}\left[A_{m} \frac{m \pi}{L} \cos \frac{m \pi x}{L} \sin Q_{m}\right]\right\} d x .
\end{aligned}
$$

Integration of the right-hand side by parts and simplification gives an expression for the rate of growth of the amplitudes,

$$
\begin{aligned}
\dot{A}_{n}=A_{n} \frac{n \pi p}{2 L} \sin 2 Q_{n}-\frac{4 f \delta x}{L p \delta t} \cos Q_{n} & \int_{0}^{L} \cos \frac{n \pi x}{L}\left\{\left|\sum_{l} A_{l} \sin \frac{l \pi x}{L} \sin Q_{l}\right|\right. \\
& \left.\times \sum_{m}\left[A_{m} \frac{m \pi}{L} \cos \frac{m \pi x}{L} \sin Q_{m}\right]\right\} d x
\end{aligned}
$$


Consider first the case $f=0$. Combining equations (5.3) and (5.4) we obtain

$$
\dot{\varphi}_{n}=-\omega_{n} \sin ^{2}\left(\omega_{n} t+\varphi_{n}\right),
$$

which on integration gives

$$
\varphi_{n}=-\omega_{n} t+\tan ^{-1}\left(\omega_{n} t+C_{n}\right),
$$

where $C_{n}$ is an arbitrary constant. With this, then

$$
\dot{A}_{n}=\omega_{n} A_{n}\left[\frac{\omega_{n} t+C_{n}}{1+\left(\omega_{n} t+C_{n}\right)^{2}}\right] \text {. }
$$

Thus

$$
A_{n}=K_{n} \sqrt{1+\left(\omega_{n} t+C_{n}\right)^{2}}
$$

where $K_{n}$ is a second arbitrary constant.

This shows that, with $f=0$, we can expect a growth in amplitude for each component, becoming linear in time for large times. This solution is also appropriate for $f \neq 0$ when the amplitudes are small enough to neglect the non-linear term in equation (5.4). But, as the amplitudes grow, this non-linear term will eventually check the instability, bringing the system to equilibrium.

Consider now the case $f \neq 0$. By neglecting cross product terms (whose contribution is small for the significant lower frequency modes), we can write equation (5.4) in a simpler form which illustrates the manner in which fluctuations are damped,

$$
\dot{A}_{n}=A_{n} \omega_{n} \sin Q_{n} \cos Q_{n}\left\{1-\frac{4 f \delta x}{L p \delta t} \int_{0}^{L}|u| \cos ^{2} \frac{n \pi x}{L} d x\right\} .
$$

The amplitudes increase in magnitude until the velocity becomes large enough to make the bracket term small. Thus, the mean value of $\dot{A}_{n}$ approaches zero to first order and $A_{n}$ achieves its maximum value. The order in which the modes are maximized depends upon the configuration of the velocity profile and of this we can say very little at first. But the velocity magnitude must continue its growth as long as there is a single mode for which $\dot{A}_{n}>0$; thus the bracket terms become negative for many modes and these oscillations decay.

Herein, perhaps, lies the explanation of the origin of the dominant mode oscillation, which was discussed in Section 3. For consider the system at the time when there is but a single mode which remains to be maximized. The velocity is increasing in magnitude but all other frequency oscillations are declining and hence the velocity profile is approaching closer and closer to the configuration of the growing oscillation. It can be shown that the integral in equation $\left(5.4^{\prime}\right)$ is least when the velocity is composed entirely of $\frac{2 \pi n}{L}$ frequency oscillations; therefore the growing resemblance of the velocity to this frequency only serves to prolong the growth of this final mode and the decay of all other modes. Thus the end of the first phase of the process, which corresponds to the time of maximum velocity magnitude, finds a major portion of the energy of the system concentrated into a single mode; this concentration increases throughout Phase II.

To include the cross product terms and perform the analysis in general would be 
difficult; however, when a particular mode, say number $\alpha$, dominates the system to the extent that

$$
\left|\sum_{l} A_{l} \sin \frac{l \pi x}{L} \sin Q_{l}\right| \rightarrow A_{\alpha}\left|\sin \frac{\alpha \pi x}{L} \sin Q_{\alpha}\right|,
$$

then considerable simplification is possible. Making use of the "dominant-mode" assumption, which will be done for the rest of this paper, equation (5.4) can be written

$$
\begin{aligned}
& \dot{A}_{n}=A_{n} \omega_{n} \sin Q_{n} \cos Q_{n}-\frac{4 f \delta x}{L p \delta t} \cos Q_{n} A_{\alpha}\left|\sin Q_{\alpha}\right| \\
& \times \sum_{m} \psi_{m} \int_{0}^{L}\left|\sin \frac{\alpha \pi x}{L}\right| \cos \frac{m \pi x}{L} \cos \frac{n \pi x}{L} d x
\end{aligned}
$$

where

$$
\psi_{m}=\frac{m \pi}{L} A_{m} \sin Q_{m}
$$

Now

$$
\int_{0}^{L}\left|\sin \frac{\alpha \pi x}{L}\right| \cos \frac{m \pi x}{L} \cos \frac{n \pi x}{L} d x=\frac{1}{\alpha \pi} \sum_{i=1}^{\alpha}\left[\frac{\cos i\left(\frac{m+n}{\alpha}\right)^{\pi}}{1-\left(\frac{m+n}{\alpha}\right)^{2}}+\frac{\cos i\left(\frac{m-n}{\alpha}\right) \pi}{1-\left(\frac{m-n}{\alpha}\right)^{2}}\right]
$$

and

$$
\sum_{i=1}^{\alpha} \cos i\left(\frac{m \pm n}{\alpha}\right) \pi=\left\{\begin{array}{lr}
\alpha, & \text { for } \frac{m \pm n}{\alpha}=k, \text { an integer } \\
0, & \text { otherwise. }
\end{array}\right.
$$

Now, in the machine calculations, it was observed that the even modes never contributed significantly to the fluctuation energy. This is reasonable to expect, since the equations with $f=0$ conserve symmetry and initially only odd modes were present; by the time even modes could couple in significantly, equilibrium of Phase II type had been achieved and further even-mode growth was slowed to second order. Thus, with only odd modes considered, the sum in equation (5.7) can be written

$$
\begin{aligned}
& S_{n} \equiv \sum_{m} \psi_{m} \int_{0}^{L}\left|\sin \frac{\alpha \pi x}{L}\right| \cos \frac{m \pi x}{L} \cos \frac{n \pi x}{L} d x=\frac{L}{\pi}\left[\psi_{n}-\frac{\psi_{|2 \alpha-n|}}{3}-\frac{\psi_{2 \alpha+n}}{3}-\frac{\psi_{|4 \alpha-n|}}{15}\right. \\
& \left.-\frac{\psi_{4 \alpha+n}}{15}-\cdots+\frac{\psi_{|2 q \alpha-n|}}{1-4 q^{2}}+\frac{\psi_{2 q \alpha+n}}{1-4 q^{2}}-\cdots\right] .
\end{aligned}
$$

Now, when $n=\alpha$, the dominant mode, only the first two terms in the sum contribute significantly to $S_{\alpha}$. Thus

$$
S_{\alpha} \rightarrow \frac{2}{3} \frac{L}{\pi} \psi_{\alpha}=\frac{2}{3} \alpha A_{\alpha} \sin Q_{\alpha}
$$

When $n \neq \alpha$, we will assume that $S_{n}$ can be limited to its first term. This assumption 
will be justified presently, at least for the significant lower-number modes, i.e., for $n<2 \alpha$. With this assumption the system of equations (5.7) can be written

$$
\begin{aligned}
& \dot{A}_{\alpha}=A_{\alpha} \omega_{\alpha} \sin Q_{\alpha} \cos Q_{\alpha}\left\{1-\frac{2}{3} \frac{4 f \delta x}{\pi p^{2} \delta t} A_{\alpha}\left|\sin Q_{\alpha}\right|\right\} \\
& \dot{A}_{n}=A_{n} \omega_{n} \sin Q_{n} \cos Q_{n}\left\{1-\frac{4 f \delta x}{\pi p^{2} \delta t} A_{\alpha}\left|\sin Q_{\alpha}\right|\right\}, \quad n \neq \alpha .
\end{aligned}
$$

Since these equations have been derived on the basis of the dominant mode assumption, which became effective at the close of Phase I of the process, they are appropriate for a description of quasi-equilibrium and equilibrium conditions, that is, Phases II and III. Without solving them we may notice their late-time properties. The rate of change of all the modes depends upon the magnitude of $A_{\alpha}$, which will continue to increase, on the average, as long as the bracket term in the first equation is positive. Before this growth stops, however, the bracket term in the equation for $A_{n}, n \neq \alpha$, will have become negative. Thus the final, Phase III, equilibrium corresponds to

$$
\begin{aligned}
& A_{\alpha} \rightarrow \frac{3}{2} \frac{\pi p^{2} \delta t}{4 f \delta x\left\langle\left|\sin Q_{\alpha}\right|\right\rangle}=\frac{3 \pi^{2} p^{2} \delta t}{16 f \delta x} \\
& A_{n} \rightarrow 0
\end{aligned}
$$$$
n \neq \alpha \text {, }
$$

where $\langle>$ signifies an average over time.

Phase II is that period, following the initial amplitude growth, when the system is approaching this single frequency oscillation. Its duration varies considerably from problem to problem and is, as shall be demonstrated, largely dependent upon the number of the dominant mode. Furthermore, the number of the dominant mode increases with system size, so that ultimately the rate of energy concentration depends upon the system size. This fact is very much in evidence in the kinetic energy profiles of Figure 1. The 6 and 12 cell systems, for which Fourier decompositions indicate the lowest frequency mode is dominant, pass rapidly from initial damping to the Phase III equilibrium condition of a uniform amplitude, single frequency oscillation. But the larger systems, which are dominated by higher frequency modes, exhibit profiles indicative of composite frequency oscillations. Furthermore, the amplitude of these kinetic energy curves increases with time indicating a slow growth in the magnitude of the dominant mode at the expense of the secondary modes.

The variation in the calculation time required to attain a true dominant mode distribution of energy and the dependence of this calculation time upon system size are apparent from Table 1, which shows the amplitude of various modes at the completion of the machine runs. This completion time is quite arbitrary in that, although all the problems have passed the stage of initial amplitude growth, there is a marked contrast in the amount of energy concentration which has taken place. The 12 cell system is the only one in which essentially all the energy of the system has been concentrated in one mode. The other problems, one of which was run considerably longer than the 12 cell problem, all contain secondary modes of significant amplitude.

We have not been able to predict a priori which mode will dominate nor to 
TABLE 1

Amplitude of significant modes at completion of machine runs for several problems

\begin{tabular}{|c|c|c|c|c|c|}
\hline \multirow{2}{*}{ Mode No. } & \multicolumn{5}{|c|}{$\begin{array}{l}\text { Number of Cells in System } \\
\text { (Duration of Problem in Time Cycles) }\end{array}$} \\
\hline & $\begin{array}{c}12 \\
(9200)\end{array}$ & $\begin{array}{c}30 \\
(9150)\end{array}$ & $\begin{array}{c}40 \\
(13,700)\end{array}$ & $\begin{array}{c}65 \\
(9700)\end{array}$ & $\begin{array}{c}80 \\
(9900)\end{array}$ \\
\hline 1 & .140 & - & .015 & - & - \\
\hline 3 & $(.002)$ & .023 & .014 & .006 & - \\
\hline j & .000 & .073 & .051 & .013 & - \\
\hline 7 & .000 & .107 & .121 & .022 & 一 \\
\hline 9 & $(.001)$ & .024 & .011 & .032 & .007 \\
\hline 11 & $(.001)$ & $(.009)$ & .001 & .048 & .028 \\
\hline 13 & & $(.002)$ & .004 & .108 & .109 \\
\hline 15 & & $(.004)$ & .001 & .033 & .032 \\
\hline 17 & & $(.006)$ & $(.002)$ & .015 & .033 \\
\hline 19 & & $(.010)$ & $(.002)$ & .032 & .036 \\
\hline 21 & & $(.011)$ & $(.003)$ & $(.003)$ & $(.009)$ \\
\hline 23 & & $(.007)$ & $(.001)$ & $(.002)$ & $(.007)$ \\
\hline 25 & & $(.005)$ & $(.001)$ & $(.002)$ & $(.006)$ \\
\hline 27 & & $(.003)$ & $(.002)$ & $(.002)$ & - \\
\hline 29 & & $(.002)$ & .000 & $(.003)$ & - \\
\hline
\end{tabular}

The amplitudes shown in parenthesis are averages of highly oscillating values.

explain why the number of the dominant mode increases with the number of cells in the system; but we can show qualitatively why the secondary mode amplitudes recede more slowly when the dominant mode wave number increases. For this purpose it seems appropriate to make use of equations (5.8), even though the system has not yet reached a true dominant mode condition. They are considered applicable because the amplitude of the dominant mode is so much larger than the secondary mode amplitudes that condition (5.6) should be valid. Simplifying equations (5.8) we write

where

$$
\begin{aligned}
& \dot{A}_{\alpha}=A_{\alpha} \omega_{\alpha}\left(1-\frac{2}{3} g A_{\alpha}\right) \\
& \dot{A}_{n}=A_{n} \omega_{n}\left(1-g A_{\alpha}\right)
\end{aligned}
$$

$$
g=\frac{4 f \delta x\left\langle\left|\sin Q_{\alpha}\right|\right\rangle}{\pi p^{2} \delta t}
$$

Solving the first equation, we obtain

$$
A_{\alpha}=\frac{R e^{\omega_{\alpha} t}}{1+\frac{2}{3} g R e^{\omega_{\alpha} t}},
$$

where $R$ is an arbitrary constant. Substituting this expression for $A_{\alpha}$ into the second equations yields a solution for $A_{n}$,

$$
A_{n}=K_{n}\left[\frac{e^{\omega_{\alpha} t}}{\left(1+\frac{2}{3} k R e^{\omega_{\alpha} t}\right)^{3 / 2}}\right]^{n / \alpha},
$$

where $K_{n}$ is another arbitrary constant. 
Notice that as $t \rightarrow \infty$,

$$
\begin{aligned}
& A_{\alpha} \rightarrow \frac{3}{2 g} \\
& A_{n} \rightarrow 0,
\end{aligned}
$$

so that the final equilibrium solution is the same as before. But equation (5.10) indicates why this final solution is delayed as the value of $\alpha$ increases. When $\alpha=1$, as in the 12 cell problem, the smallest possible value of the exponent is three so that all the secondary modes lose amplitude rapidly; but for larger values of $\alpha$ there exist modes for which this exponent is approximately one, so that the decay of these amplitude curves is much more gradual.

Likewise this equation demonstrates why, for a fixed value of $\alpha$, the high frequency vibrations are damped much more rapidly than the lower frequency ones, substantiating our assumption that the high frequency contributions to $S_{n}$ could be neglected when $n<2 \alpha$. This rapid decay of the high frequency modes is apparent in Table 2, which, for a system of 40 cells, compares the amplitude of significant modes at an earlier Phase II time with those at problem completion time as listed in Table 1. Notice also, in Table 2, the growth of the dominant mode amplitude.

Now as the amplitude of these high frequency vibrations begins to change rapidly, their phase angles once again assume a strong time dependency as a result of the condition expressed in equation (5.3). These complications are made apparent in the computer results by rather erratic variations in the amplitude and period of these high frequency oscillations at late times. The irregular character of these oscillations is indicated in Table 1 by parentheses around their average amplitude value.

On the other hand, in the larger system, the lower frequency oscillations are extremely uniform in both amplitude and period at the time that these computer runs were completed. This would indicate that the dominant mode amplitude has not as yet changed much from the value which would make $\dot{A}_{n}=0$ in Eq. (5.8), i.e.,

$$
A_{\alpha} \approx \frac{\pi^{2} p^{2} \delta t}{8 f \delta x}
$$

And, indeed, the dominant mode amplitudes in Table 1 do not vary much from this value; the greatest variation is $13 \%$.

Since this rather slow rate of growth of the dominant mode is somewhat at odds with what one would predict from equations (5.8), it is perhaps worthy of

TABLE 2

Amplitude of Significant Modes at Early and Later Equilibrium Times for a System of 40 Cells

\begin{tabular}{c|c|c|c|c|c|c|c}
\multicolumn{1}{c|}{ Time } & \multicolumn{8}{c}{ Mode Number } \\
\hline & 1 & 3 & 5 & 7 & 9 & 11 & 13 \\
\hline 7,300 & .015 & .016 & .053 & .093 & .061 & .022 & .017 \\
13,700 & .015 & .014 & .051 & .121 & .011 & .001 & .004 \\
\hline
\end{tabular}


some additional comment. Notice in these equations that the expression for $\dot{A}_{\alpha}$ is a product of two factors,

$$
A_{\alpha} \omega_{\alpha} \sin Q_{\alpha} \cos Q_{\alpha}
$$

and

$$
\left\{1-\frac{2}{3} \frac{4 f \delta x}{\pi p^{2} \delta t} A_{\alpha}\left|\sin Q_{\alpha}\right|\right\} .
$$

The bracket term is positive for the Phase II dominant mode amplitudes given by equation (5.11) and hence any slowing down of the rate of growth must result from the first term. This could happen if the first term was approaching an oscillating function of time, in which case the product of the two terms would oscillate and $A_{\alpha}$ would experience alternating periods of growth and decay. As this first term becomes a sinusoidal function, $\dot{A}_{\alpha}$ vanishes on the average and the system reaches final equilibrium.

There is some reason to believe that the final equilibrium state, which these machine problems are approaching, is characterized more by the sinusoidal nature of this first term than by the vanishing of the bracket term. Some evidence for this is given by the amplitude of the dominant mode of the 12 cell system at problem completion time. Both the extreme concentration of energy into a single mode, evident in Table 1, and the uniform kinetic energy amplitude of Figure 1 indicate that this 12 cell problem is at least very close to a final equilibrium state. And yet the final dominant mode amplitude for this problem is .140, which is only $76 \%$ of the value which would make the bracket term in equation (5.8) vanish. Hence the constancy of the dominant mode amplitude must result from the vanishing on the average of the first term.

This matter will be referred to again in the discussion of final kinetic energy predictions in Section 6 .

6. Predictions. Consider, now, an estimate of the total kinetic energy of the system on the basis of this Phase II dominant mode amplitude (equation (j.11)). The kinetic energy in the $j$ th cell is given by

$$
K E_{j}=\frac{m}{2} \sum_{m} \sum_{n} A_{m} \sin \frac{m \pi j}{N} \sin Q_{m} A_{n} \sin \frac{n \pi j}{N} \sin Q_{n},
$$

so that, with $m=1$

and

$$
\left\langle K E_{j}\right\rangle=\frac{1}{4} \sum_{n} A_{n}{ }^{2} \sin ^{2} \frac{n \pi j}{N}
$$

$$
\langle K E\rangle=\sum_{j}\left\langle K E_{j}\right\rangle=\frac{N}{8} \sum_{n} A_{n}{ }^{2} .
$$

Assuming that the dominant mode contains essentially all of the energy in the system, this gives

$$
\langle K E\rangle=\frac{N}{8}\left[\frac{\pi^{2} p^{2} \delta t}{8 f \delta x}\right]^{2} .
$$


This prediction is shown as the middle line in Figure 2. The agreement is considerably better (at this stage) than that obtained in the first analysis; any discrepancy is a measure of the actual strength of the neglected secondary modes.

But from this intermediate stage of equilibrium we expect the kinetic energy profile to rise rather slowly as the dominant mode amplitude approaches its asymptotic value given by equation (5.9). Since the asymptotic dominant mode amplitude is $\frac{3}{2}$ the value used in the kinetic energy determination above, we might expect that the kinetic energy of the system would eventually attain a level $\frac{9}{4}$ higher than this intermediate plane. However we expect this growth will be tempered by the fact that, as $\dot{A}_{\alpha}$ becomes small, the first factor in the expression for $\dot{A}_{\alpha}$ will become sinusoidal so that $\dot{A}_{\alpha}$ will become an oscillating function of time in equation (5.8). The final kinetic energy level should then lie at some intermediate plateau, probably not far removed from the prediction made by the first analysis. Unfortunately, a prohibitive amount of machine time would be required to enable one of these large systems to reach this ultimate goal.

It is also possible to obtain a fairly accurate estimate of the frequency of the dominant mode at equilibrium. When the system has attained a true dominant mode energy distribution, indicative of Phase III equilibrium, and the dominant mode is approaching its limiting value, then its frequency should be approaching the natural frequency, since $\dot{\varphi}_{\alpha} \rightarrow 0$ with $\dot{A}_{\alpha}$, according to equation (5.3). In the 12 cell system, which is a case of this type, the frequency of the first mode differs by less than two per cent from its natural frequency.

But for a system which has only attained the intermediate stage of equilibrium, a prediction is somewhat more difficult to obtain. The relationship between the amplitude and phase of the dominant mode in such a system is described by equations (5.8) and (5.3),

$$
\begin{gathered}
\dot{A}_{\alpha}=\frac{1}{2} A_{\alpha} \sin 2 Q_{\alpha}\left\{\omega_{\alpha}-Z A_{\alpha}\left|\sin Q_{\alpha}\right|\right\}, \quad Z=\frac{8 \alpha f \delta x}{3 L p \delta t} \\
\dot{A}_{\alpha} \sin Q_{\alpha}+A_{\alpha}\left(\dot{Q}_{\alpha}-\omega_{\alpha}\right) \cos Q_{\alpha}=0 .
\end{gathered}
$$

Since the experimental evidence indicates that the amplitude and period of the dominant mode vary quite slowly at this stage, let us set

$$
\begin{aligned}
& A_{\alpha}=K+\zeta \\
& Q_{\alpha}=\Omega t+\epsilon
\end{aligned}
$$

in these equations, where $\zeta$ and $\epsilon$ are higher order correction terms. Neglecting higher order terms, this gives

$$
\begin{gathered}
\dot{\zeta}=\frac{1}{2} K \sin 2 \Omega t\left(\omega_{\alpha}-Z K|\sin \Omega t|\right) \\
\dot{\zeta} \sin \Omega t+K\left(\Omega-\omega_{\alpha}+\dot{\epsilon}\right) \cos (\Omega t+\epsilon)=0
\end{gathered}
$$

or

$$
\sin 2 \Omega t\left(\omega_{\alpha}-Z K|\sin \Omega t|\right)+\Omega-\omega_{\alpha}+\dot{\epsilon}=0 .
$$

Now average over time and assume that $\langle\dot{\boldsymbol{\epsilon}}\rangle=0$ to get

$$
\Omega=\frac{\omega_{\alpha}}{2}+\frac{4 Z K}{3 \pi} \text {. }
$$


TABLE 3

Comparison Between Observed and Predicted Dominant Mode Frequencies at Intermediate Stage of Equilibrium

\begin{tabular}{c|c|c|c|c} 
& \multicolumn{4}{|c}{ Number of Cells } \\
\hline & 30 & 40 & 65 & 80 \\
\hline Observed Frequency & .427 & .331 & .370 & - \\
$\therefore \Omega=1 \frac{7}{8} \omega_{\alpha}$ & .439 & .329 & .375 & .323 \\
\hline
\end{tabular}

For $K$ use the Phase II dominant mode amplitude given by equation (5.11). Then

$$
\Omega=\frac{\omega_{\alpha}}{2}+\frac{4}{3 \pi} \frac{8 \alpha f \delta x}{3 L p \delta t} \frac{\pi^{2} p^{2} \delta t}{8 f \delta x}=\frac{17}{18} \omega_{\alpha} .
$$

Table 3 shows a comparison of this predicted dominant mode frequency with the observed results for problems at this intermediate stage of equilibrium.

7. Summary. The instability inherent in the coupled non-linear difference equations (2.2) gives rise to rapidly growing fluctuations of velocity when steady state conditions are perturbed. These instabilities grow rapidly for a time but infinite growth is curbed by the dissipative properties of the equations which damp the fluctuations to a preliminary equilibrium state. This period of development is characterized by a modal exchange of energy which culminates in the emergence of a single dominant mode of oscillation in the system. A final equilibrium state is attained when the secondary mode amplitudes have all decayed to insignificance; henceforth the system oscillates at the natural frequency of the dominant mode with a constant amplitude.

University of California

Los Alamos Scientific Laboratory

Los Alamos, New Mexico

1. M. W. Evans \& F. H. Harlow, "The Particle-In-Cell Method for Hydrodynamic Calculations," Los Alamos Scientific Laboratory Report LA-2139, November 1957.

2. B. J. DALY, "The Bounding of Instabilities of the PIC Difference Equations," Los Alamos Scientific Laboratory Report LA-2414, December 1962.

3. N. KRYLOFF \& N. BogoliubofF, Introduction to Non-Linear Mechanics, Annals of Mathematics Studies, Number 11, Princeton University Press, 1947. 